\title{
An Efficient Nomogram to Predict Overall Survival of Patients with Pediatric Ewing's Sarcoma: A Population-Based Study
}

\author{
Ke-Na Dai ${ }^{1}$ \\ A-Bing $\mathrm{Li}^{2}$ \\ 'Department of Pediatrics; Ningbo \\ Medical Center Lihuili Hospital, Ningbo, \\ Zhejiang, 315040, People's Republic of \\ China; ${ }^{2}$ Department of Orthopedics, \\ Ningbo Yinzhou Second Hospital, \\ Ningbo, 3/5100, Zhejiang, People's \\ Republic of China
}

Background: The objective of our study was to develop and validate a nomogram to predict the overall survival (OS) of patients with pediatric Ewing's sarcoma (PES).

Methods: Age, gender, race, tumor stage, tumor size, tumor site, treatment method, and survival time were collected from patients diagnosed with PES between 2004 and 2016 from the Surveillance, Epidemiology, and End Results (SEER) database. A total of 772 patients were randomly allocated to a training dataset $(n=579)$ and a validation dataset $(n=193)$. Then, univariate and multivariate analyses were performed to determine the prognostic effect of the selected variables. A nomogram was constructed to estimate the OS and it was further assessed using the concordance index (C-index), calibration curves, and receiver operating characteristic (ROC).

Results: Age, race, tumor size, and tumor stage were included in the nomogram. The $\mathrm{C}$-index was 0.77 in the $\mathrm{OS}$ for the training dataset. The $\mathrm{C}$-index for the validation dataset of the OS prediction was 0.75 . Calibration plots and ROC curves showed excellent predictive accuracy.

Conclusion: Age, race, tumor stage, and tumor size were independent prognostic factors for patients with PES. The nomogram showed an accurate and reliable prognostic performance for PES patients.

Keywords: nomogram, prognosis, SEER program, pediatric Ewing's sarcoma

\section{Introduction}

Ewing's sarcoma (ES), first described by James Ewing, is a malignant bone or softtissue tumor that mainly affects children, adolescents, and young adults. ${ }^{1,2} \mathrm{ES}$ is classically described under small round cell tumor which occurs predominantly in leg bones and pelvis. ${ }^{3}$ In the United States, the population-adjusted incidence of ES during the 30-year study period did not significantly change and remained at the rate of about 2.9/100,000 per year. ${ }^{4}$ Previous researches have described prognostic variables for ES. ${ }^{3,5-9}$ Although, in recent years, the survival rate of ES patients has remarkably improved owing to the progress in the treatment methods, the mentioned rate is still low in patients with advanced cancer and the overall therapeutic effect is unsatisfactory as well. ${ }^{10,11}$ Thus, it is highly essential to carry out further research on early diagnosis and management of ES to improve the survival rates and quality of life of ES patients.

Given the clinical manifestations of ES, it is urgent to develop a prognostic model to assist clinicians to more precisely estimate prognosis of ES patients.
Correspondence: A-Bing $\mathrm{Li}$ Department of Orthopedics, Ningbo Yinzhou Second Hospital, Ningbo, Zhejiang, 315100 , People's Republic of China

Tel +86-I508855762I

Email bing19890807@hotmail.com 
Nomogram for ES is suggested as an efficient statistical tool to predict a patient's survival. ${ }^{9-12}$ A nomogram is considered a new prognostic tool compared with other staging systems. Although there are nomograms for Ewing's sarcoma, ${ }^{9,11}$ no nomogram has been reported for pediatric Ewing's sarcoma (PES). ES is more common in children but relatively rare in adults. Age is an important prognostic factor. Adults with ES have a poorer prognosis compared to children. ${ }^{13,14}$ In addition, the existing treatment-related factors are significantly different between children and adults. ${ }^{15}$ So, the prognosis of pediatric and adult diseases needs to be addressed separately. Therefore, the present study aimed to develop a nomogram to estimate the overall survival (OS) of PES patients.

\section{Methods}

\section{Patient Selection}

The Surveillance, Epidemiology, and End Results (SEER) database covers approximately $30 \%$ of the US population which could collect sufficient cases for investigation. ${ }^{11}$ PES patients diagnosed between 2004 and 2016 were included in this study. The inclusion criteria were as follows: (1) patients with a diagnosis of PES between 2004 and 2016; (2) histological type according to the International Classification of Diseases for Oncology, 3rd edition (ICD-O-3) code 9260/3; (3) tumor site was selected as C40.0-C41.9; and (4) age < 20 years. Exclusion criteria were: (1) incomplete clinicopathological information (tumor stage, tumor size); (2) patients without chemotherapy; and (3) multiple primary cancers or extraosseous Ewing sarcoma. As SEER is a public research database, approval from the ethical board and informed consent from individual patients were not required.

\section{Variables}

The variables extracted from the SEER program database included age, gender, race, tumor stage, tumor size, tumor site, treatment method, and survival time. Age was stratified into two groups ( $\leq 14$, and $>14$ years $)$, the tumor size was stratified into two groups ( $\leq 8$ and $>8 \mathrm{~cm}$ ) using the "Collaborative Stage (CS) tumor size (2004-2015)" variable in the database, and the race was stratified into two groups (white and back + others). To ensure a coherent cancer staging classification across the study period, the "SEER historical stage" was employed. It included three clinically relevant categories: localized, regional, or distant disease. According to the 2018 version of the Summary
Stage Manual provided by SEER (https://seer.cancer.gov/ tools/ssm/), localized indicated that the tumor was confined to the cortex of the bone or extended beyond the cortex to the periosteum (no break in periosteum), regional indicated that the tumor extended beyond periosteum to the surrounding tissues, including adjacent skeletal muscle(s), distant indicated that tumor has spread to parts of the body remote from the primary tumor. The information on radiotherapy and surgery was based on the variables "RX Summ-Surg Prim Site (1998+)" and "Radiation recode". The anatomic location was reclassified as either axial skeleton (pelvis, spine, and ribs) or appendicular skeleton (long and short bones of the upper and lower extremities).

\section{Statistical Methods}

Patients were randomly separated into a training dataset (n $=579)$ and a validation dataset $(\mathrm{n}=193)$ in a $3: 1$ ratio to construct and validate the nomogram. The chi-square test and Fisher exact test were used to analyze the differences between groups. To construct a nomogram, at first, all the variables were enrolled in the univariate Cox proportional hazards analysis for the OS. Secondly, factors with $\mathrm{p}<0.1$ in univariate analysis were further analyzed in a multivariate Cox proportional hazards model. Thirdly, a backward stepwise technique was used to select the prognostic elements authenticated in the multivariate analysis by acquiring the minimum Akaike information criterion (AIC) value. The AIC is an objective tool for selecting various models ${ }^{16}$ and a lower AIC value suggests a preferable model. Finally, factors that have the smallest AIC value were included in the final model and were used to construct a nomogram. The concordance index (C-index) and receiver operating characteristic (ROC) were used to evaluate the prognostic nomogram performance. Calibration curves were constructed to determine whether the predicted survival was consistent with the actual survival. Statistical software R (version 3.34, http://www.r-project.org) was applied for all data analyses, and $\mathrm{p}$-value $<0.05$ indicated statistical significance.

\section{Results \\ Baseline Characteristics}

A total of 772 PES patients were enrolled in this study and randomly assigned to the training dataset $(n=579)$ and validation dataset $(n=193)$ to construct and validate a nomogram, respectively. Of these patients, 472 
$(61.14 \%)$ were male and $300(38.86 \%)$ were female; 504 $(65.28 \%)$ and $268(34.72 \%)$ were aged $\leq 14$ and $>14$ years old, respectively. Besides, $38.6 \%$ of them were diagnosed as localized, $32.12 \%$ as regional, and $29.27 \%$ as a distant disease; $46.5 \%$ of the cases were categorized as an appendicular skeleton and $53.5 \%$ as an axial skeleton. The majority of the patients were white $(88.21 \%)$. A total of 486 patients $(62.95 \%)$ underwent surgery, of whom $24.48 \%$ received radiation therapy. No significant difference was found between training and validation datasets concerning patients' demographic and pathological features (Table 1).

The results of the univariate analysis suggested that age, race, tumor stage, tumor size, tumor location, and surgery were significantly correlated with the OS (Table 2). The results of multivariate regression analysis revealed that only four factors: age, race, tumor size, tumor stage were independent prognostic factors for the OS (Table 2).

\section{Establishment and Verification of the Nomogram for the OS and CSS}

Independent prognostic factors were chosen using a backward stepwise method with the AIC to reduce information drop. After selection, age, race, tumor size, and tumor stage were included in the final model to construct a nomogram (Figure 1). The nomogram endows each predictor with a point (Table 3 ). The $\mathrm{C}$-index was 0.77 (95\% CI, 0.74 to 0.80$)$ in the OS for the training dataset, and the C-index was 0.75 (95\% CI, 0.68 to 0.82 ) in the $\mathrm{OS}$ for the validation dataset. For the training dataset, the 3, and 5-year AUC values of the nomogram of the OS were 0.82 and 0.81 , respectively (Figure 2). For the validation dataset, the 3- and 5-year AUC values of the nomogram of the OS were 0.77 and 0.76 , respectively (Figure 3). Calibration plots for 3- and 5-year OS demonstrated good consistency between actual survival and nomogram prediction (Figures 4 and 5).

Table I Patient Demographics and Pathological Characteristics

\begin{tabular}{|c|c|c|c|c|}
\hline Characteristics & Total (n,\%) & Training Cohort $(\mathrm{n}, \%)$ & Validation Cohort $(n, \%)$ & $\mathbf{P}$ \\
\hline Age & & & & 0.26 \\
\hline$\leq 14$ & $504(65.28)$ & $385(66.49)$ & $119(61.66)$ & \\
\hline$>14$ & $268(34.72)$ & $194(33.5 I)$ & $74(38.34)$ & \\
\hline Sex & & & & 0.35 \\
\hline Male & $472(61.14)$ & $348(60.1)$ & $124(64.25)$ & \\
\hline Female & $300(38.86)$ & $23 I(339.9)$ & $69(35.75)$ & \\
\hline Race & & & & 0.95 \\
\hline Black + Other & $91(11.79)$ & $69(11.92)$ & $22(11.4)$ & \\
\hline White & $68 I(88.2 I)$ & $510(88.08)$ & $|7|(88.6)$ & \\
\hline Seerstage & & & & 0.71 \\
\hline Localized & $298(38.6)$ & $221(38.17)$ & $77(39.9)$ & \\
\hline Regional & $248(32.12)$ & |84(31.78) & $64(33.16)$ & \\
\hline Distant & $226(29.27)$ & $174(30.05)$ & $52(26.94)$ & \\
\hline Tumor site & & & & 0.53 \\
\hline Appendicular & $359(46.5)$ & $265(45.77)$ & $94(48.7)$ & \\
\hline Axial & $4 \mid 3(53.5)$ & $314(54.23)$ & $99(51.3)$ & \\
\hline Tumor size & & & & 0.90 \\
\hline$\leq 8 \mathrm{~cm}$ & $459(59.46)$ & $343(59.24)$ & $116(60.1)$ & \\
\hline$>8 \mathrm{~cm}$ & $313(40.54)$ & $236(40.76)$ & $77(39.9)$ & \\
\hline Surgery & & & & 0.50 \\
\hline Yes & $486(62.95)$ & $369(63.73)$ & $117(60.62)$ & \\
\hline No & $286(37.05)$ & $210(36.27)$ & $76(39.38)$ & \\
\hline Radiation & & & & 0.051 \\
\hline No & $583(75.52)$ & $448(77.37)$ & $135(69.95)$ & \\
\hline Yes & $189(24.48)$ & $|3|(22.63)$ & $58(30.05)$ & \\
\hline
\end{tabular}


Table 2 Univariate and Multivariate Analyses of Overall Survival in the Training Set

\begin{tabular}{|c|c|c|c|c|}
\hline \multirow[t]{2}{*}{ Characteristics } & Univariate Analysis & \multirow[t]{2}{*}{$\mathbf{P}$} & Multivariate Analysis & \multirow[t]{2}{*}{$\mathbf{P}$} \\
\hline & HR(95\% Cl) & & HR(95\% Cl) & \\
\hline \multicolumn{5}{|l|}{ Age } \\
\hline$\leq 14$ & Reference group & & Reference group & \\
\hline$>14$ & $1.75(1.28-2.38)$ & $<0.05$ & $1.24(I .02-1.72)$ & $<0.05$ \\
\hline \multicolumn{5}{|l|}{ Sex } \\
\hline Male & Reference group & & & \\
\hline Female & $0.9(0.66-1.24)$ & 0.52 & & \\
\hline \multicolumn{5}{|l|}{ Race } \\
\hline Black + Other & Reference group & & Reference group & \\
\hline White & $0.65(0.42-1)$ & 0.052 & $0.61(0.39-0.96)$ & $<0.05$ \\
\hline \multicolumn{5}{|l|}{ Seerstage } \\
\hline Localized & Reference group & & Reference group & \\
\hline Regional & $2.01(1.21-3.34)$ & 0.07 & $\mathrm{I} .5(0.89-2.5 \mathrm{I})$ & 0.126 \\
\hline Distant & $6.7(4.2-10.67)$ & $<0.05$ & $4.03(2.46-6.6 I)$ & $<0.05$ \\
\hline \multicolumn{5}{|l|}{ Tumor site } \\
\hline Appendicular & Reference group & & Reference group & \\
\hline Axial & $1.68(1.22-2.32)$ & $<0.05$ & $1.25(0.9-1.74)$ & 0.183 \\
\hline \multicolumn{5}{|l|}{ Tumor size } \\
\hline$\leq 8 \mathrm{~cm}$ & Reference group & & Reference group & \\
\hline$>8 \mathrm{~cm}$ & $4.35(3.07-6.17)$ & $<0.05$ & $3.17(2.2-4.56)$ & $<0.05$ \\
\hline \multicolumn{5}{|l|}{ Surgery } \\
\hline Yes & Reference group & & Reference group & \\
\hline No & $2.21(1.62-3)$ & $<0.05$ & $\mathrm{I} .3 \mathrm{I}(0.94-\mathrm{I} .82)$ & 0.11 \\
\hline \multicolumn{5}{|l|}{ Radiation } \\
\hline No & Reference group & & & \\
\hline Yes & $1.09(0.77-1.55)$ & 0.633 & & \\
\hline
\end{tabular}

\section{Discussion}

This study aimed to develop a nomogram for predicting the OS in PES patients. The C-index and the area under the curve (AUC) values indicated an excellent diagnostic performance in both the training and validation datasets. The calibration plots showed that the constructed nomogram accurately predicted the OS. This nomogram can be used for clinical assessment of PES patients and determination of the prognosis. It revealed that age, race, tumor size, and tumor stage affected the prognosis of PES patients.

Several studies correlated age with the prognosis of sarcomas. $3,6,8,17$ Stiller et al found that the two-year survival of ES patients who aged $<15$ years old was $55 \%$ during 1980 to 1982 , and reached $85 \%$ from 1989 to $1991 .{ }^{18}$ Linabery et al demonstrated that patients aged 15-19 years old had notably lower 5-year rates compared with their younger counterparts. ${ }^{17}$ Cotterill et al reported that tumor site and age ( $<15$ vs $\geq 15$ years old $)$ had a significant influence on event-free survival. ${ }^{3}$ In the current study, we noted that age is one of the key prognostic factors for young patients with PES.

The incidence of ES in patients of the white race was found to be the highest. The biological behavior of tumors can be markedly affected by genetic factors. ${ }^{19}$ About $80 \%$ of Europeans carry at least one copy of the variant allele 
Points

$\begin{array}{llllllllll}0 & 10 & 20 & 30 & 40 & 50 & 60 & 70 & 80 & 90\end{array}$

\section{Age}

Race

Tumorsize

Seerstage

Total Points

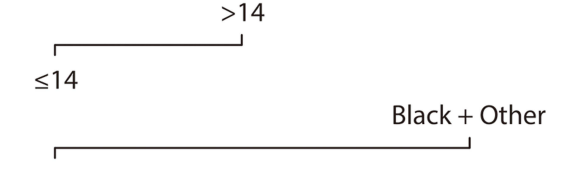

White

3-year survival

$$
\leq 8 \mathrm{~cm} \text { Regional }
$$

Localized Distant

5-year survival

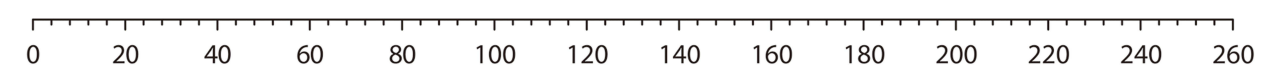

\begin{tabular}{llllllll}
\hline 0.95 & 0.9 & 0.85 & 0.8 & 0.75 & 0.7 & 0.6 & 0.5
\end{tabular}

5-year survival

\begin{tabular}{lllllll}
\hline 0.9 & 0.85 & 0.8 & 0.75 & 0.7 & 0.6 & 0.5
\end{tabular}

Figure I Nomograms for predicting the 3- and 5-year overall survival of pediatric Ewing's sarcoma. Description using nomograms: First, each feature point of the patient is assigned by plotting a vertical line to a point scale from the variable. Then, sum all the points and draw a vertical line from the total point scale to the liver metastasis axis to obtain the probability.

(EWSR1-FLI1) in their germline DNA, while this mutation is rare in African populations. ${ }^{20}$ Davenport et al and Worch et al reported that the white patients had longer survival than patients with other races, ${ }^{21,22}$ which is consistent with the results of the present research. Duchman et $\mathrm{al}^{6}$ found that patients of the black race were at a higher

Table 3 Detailed Scores of Prognostic Factors in the Overall

\begin{tabular}{|c|c|}
\hline Characteristics & OS Nomogram \\
\hline \multicolumn{2}{|l|}{ Age } \\
\hline$\leq 14$ & 0 \\
\hline$>14$ & 15.58 \\
\hline \multicolumn{2}{|l|}{ Race } \\
\hline Black + Other & 34.56 \\
\hline White & 0 \\
\hline \multicolumn{2}{|l|}{ Tumorsize } \\
\hline$\leq 8 \mathrm{~cm}$ & 0 \\
\hline$>8 \mathrm{~cm}$ & 76.72 \\
\hline \multicolumn{2}{|l|}{ Seerstage } \\
\hline Localized & 0 \\
\hline Regional & 28.64 \\
\hline Distant & 100 \\
\hline
\end{tabular}
Survival Nomograms risk of metastasis. Ethnic-based differences also play an important role in the appearance and progression of ES.

Previous studies indicated that advanced tumor stage and larger tumor size were associated with a poor OS. ${ }^{9,23}$ Lee et al pointed out that tumor size could affect survival rate, and tumor size $>8 \mathrm{~cm}$ was associated with poorer survival than that of $\leq 8 \mathrm{~cm} .{ }^{24}$ Leavey et al found that prognosis was substantially worsened in patients with tumor size $>8 \mathrm{~cm}$ and the recurrence rate of ES was correlated to the tumor size. ${ }^{7}$ Larger tumor size is more likely to be associated with metastatic disease. These previously achieved findings were consistent with the results of the current study.

ES is an aggressive primary bone tumor, predominantly influencing children and young adults. Studies reported the occurrence of ES in approximately 18.4$32 \%$ of patients with a history of metastatic disease. ${ }^{3,6}$ In the present research, 29\% of PES patients had a history of metastasis before the initial diagnosis. Esiashvili et al reported that over the past three decades, the survival rate of patients with metastases was raised. ${ }^{4}$ Although great progress has been made in the treatment of ES, the 5-year survival rate of ES patients with a history of metastasis is still insignificant. ${ }^{25,26}$ To date, little is known about risk factors related to higher odds of metastasis at initial diagnosis in ES patients. ${ }^{27}$ Our results revealed that PES 


\section{AUC of OS}

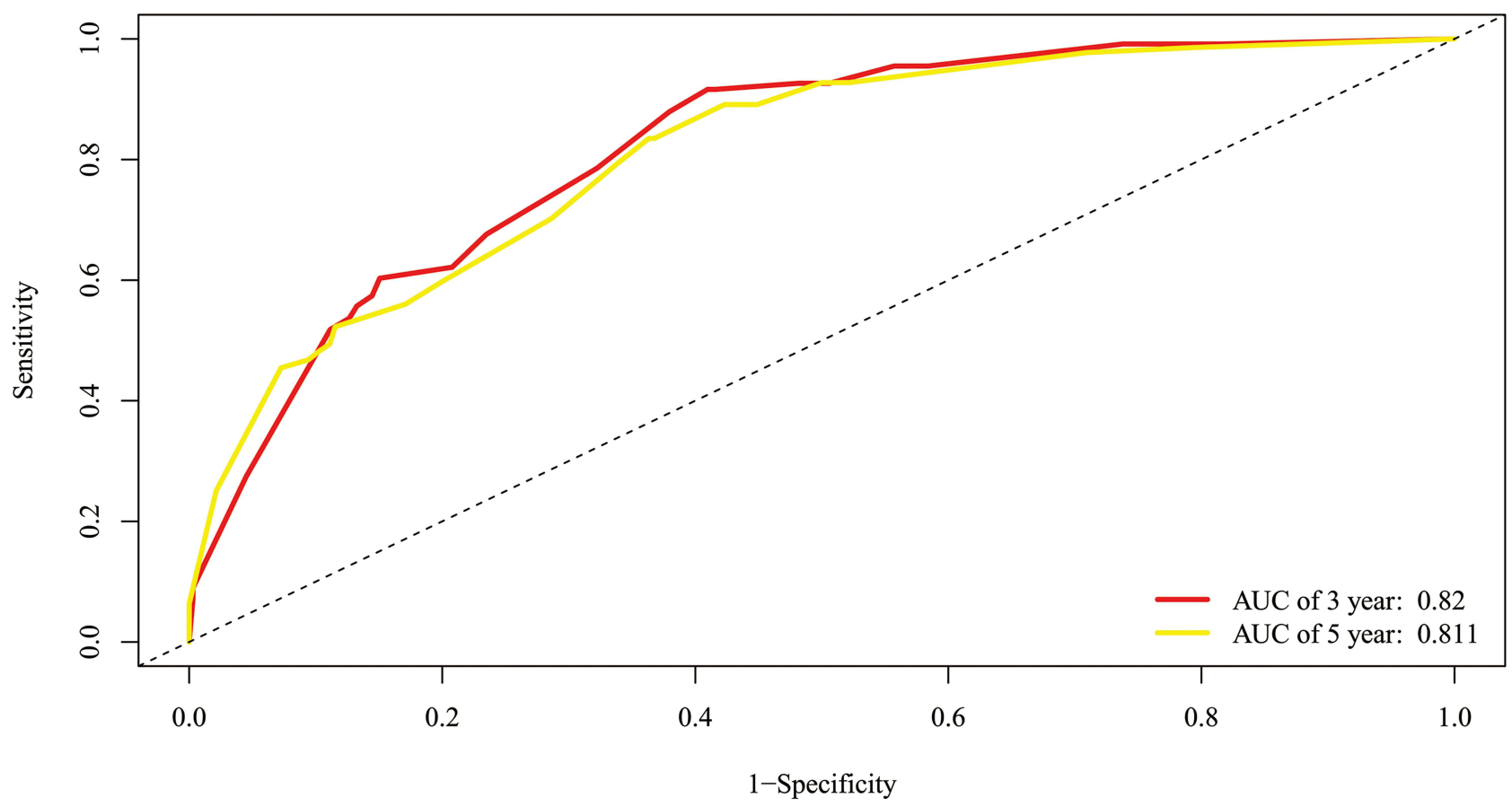

Figure 2 AUC values of ROC predicted overall survival in the training set.

\section{AUC of OS}

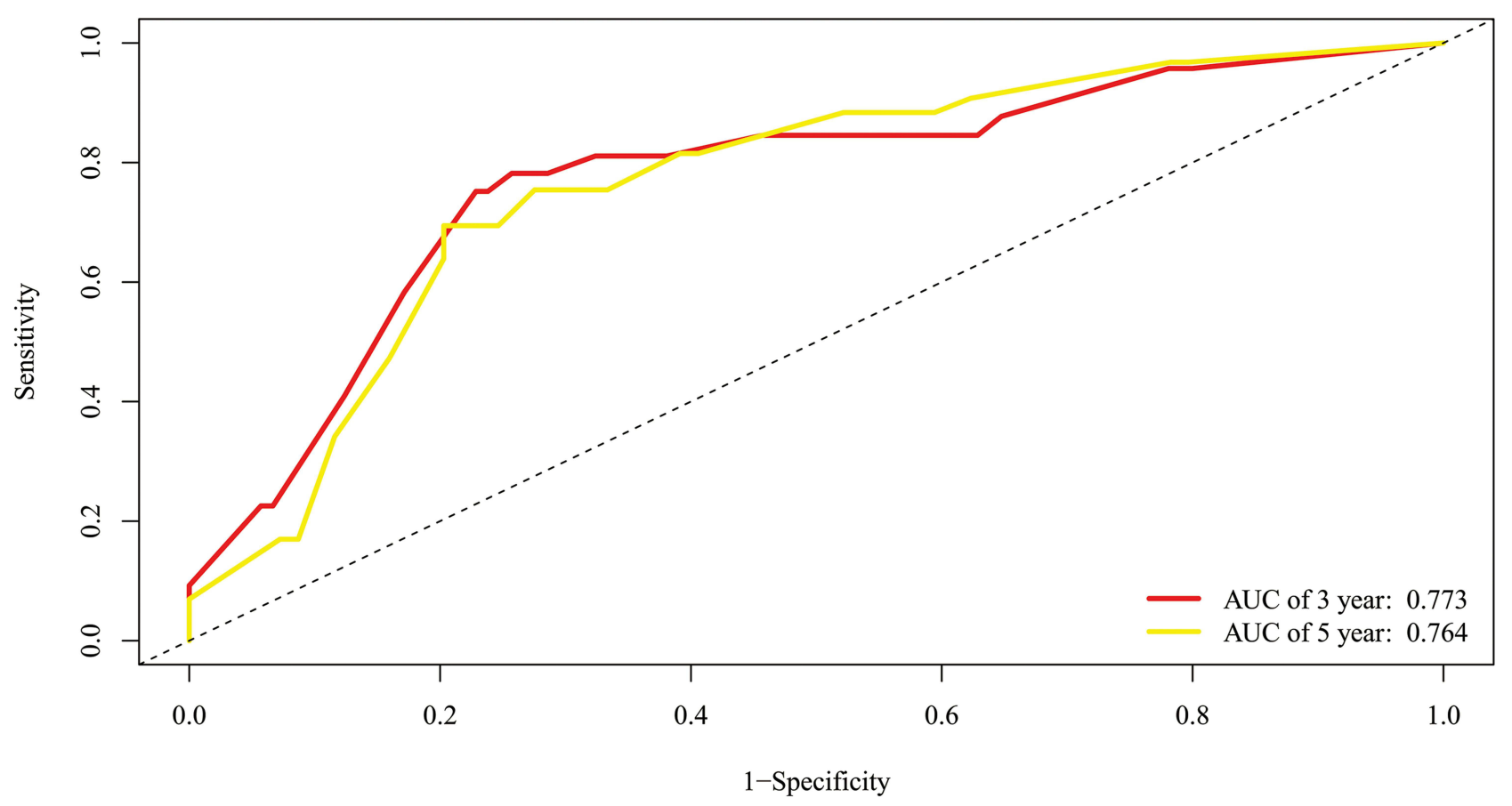

Figure 3 AUC values of ROC predicted overall survival in the validation set. 

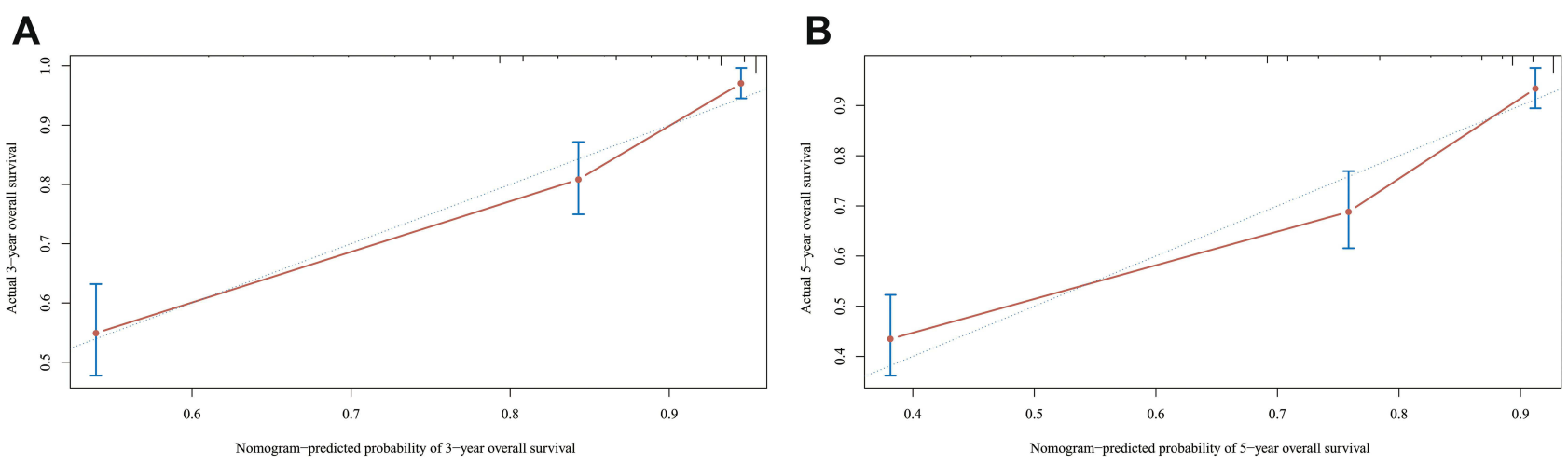

Figure 4 Training cohort calibration plot. (A) 3-year and (B) 5-year overall survival nomogram calibration curves. The 45-degree line represents an ideal match between actual survival $(y$-axis) andnomogram-predicted survival ( $x$-axis). The perpendicular line means $95 \%$ confidence intervals.

A

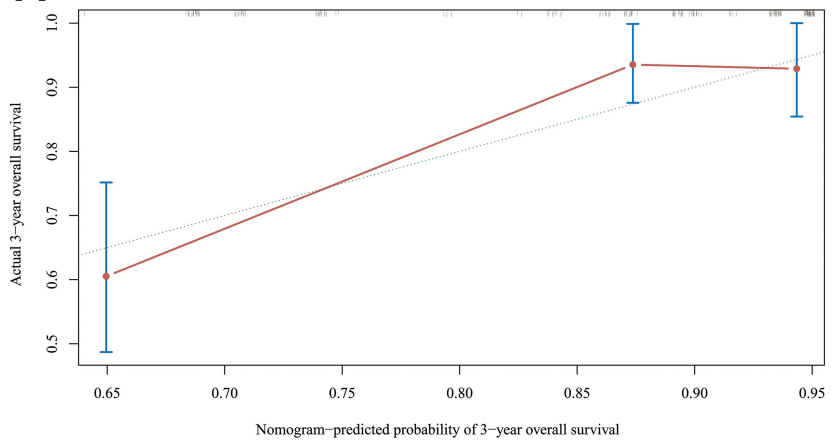

B

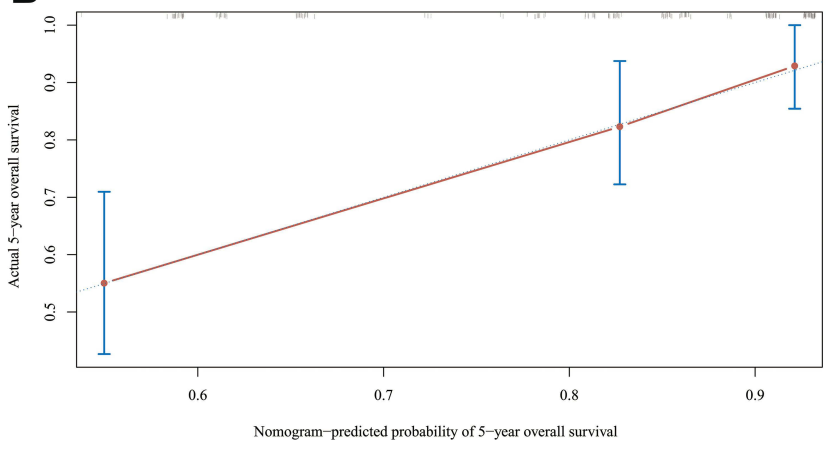

Figure 5 Validation cohort calibration plot. (A) 3-Year and (B) 5-year overall survival nomogram calibration curves. The 45-degree line represents an ideal match between the actual survival (Y-axis) and nomogram-predicted survival (X-axis). The perpendicular line means $95 \%$ confidence intervals.

patients in distant-stage had poorer OS than those with localized or regional stages. Thus, it is crucial to diagnose and treat tumors at an early stage to improve the survival rate.

The treatment approaches for ES have evolved over the recent decades. Chemotherapy, radiotherapy, and surgery have been employed in the systemic treatment of ES. Chemotherapy is the most important therapeutic method for the management of the ES. ${ }^{28,29}$ Chemotherapy is often given before local treatment. Preoperative chemotherapy can improve the efficacy of surgical resection for ES and can simultaneously restrain tumor growth. ${ }^{28}$ Surgery and radiotherapy are integral components of the treatment of localized ES. The results of our study revealed that surgery and radiation therapy were not independent prognostic factors. However, according to the findings of univariate analysis, patients who were not treated with surgery had 2.2 times higher risks of death. This may be related to the fact that the effects of local therapy depend on the tumor size, tumor site, tumor stage, and age which could act as confounders.
Using the nomogram, doctors could conveniently and precisely predict an individual patient's survival probability. An example is as follows: Consider a 15-year-old (15.58 points) white patient (0 points) with a tumor size of $6 \mathrm{~cm}$ ( 0 points) with the regional stage (28.64 points). The point total is 44.22 in the nomogram we established. The 3-year and 5-year OS predictions for this patient would be $91 \%$ and $86 \%$, respectively. In addition, a nomogram can be used to handle complex situations when there is no clear clinical guidance. The nomogram can be used to guide clinical decision-making and to evaluate overall survival, thereby facilitating a more rational allocation of medical resources. For the patients with low life expectancy, chemotherapy could be chosen because they are unlikely to benefit more from the surgery.

The present study contains several limitations. First, the retrospective nature of this study might have resulted in selection bias. Second, there is an absence of some parameters in the SEER database that are important for predicting prognoses, such as tumor markers, surgical margins, local and 
distant recurrence, regional nodal disease, and details of metastasis site. Third, although the $\mathrm{C}$-index and AUC values were promising in the validation dataset, if another dataset could be used for external validation, the results might be more reliable. Despite these shortcomings, the constructed nomogram is clinically significant, effective, and can meaningfully assist clinicians in the assessment of patients' survival.

\section{Conclusions}

Age, race, tumor stage, and tumor size are independent prognostic factors for patients with PES. Based on these variables, we constructed a nomogram to predict the OS that showed an accurate and reliable prognostic performance for PES patients.

\section{Ethics Approval and Informed Consent}

Our data from SEER database. This is a public research database. Due to the informed patient consent in SEER database is not requires, it considered that ethical approval is not needed.

\section{Acknowledgments}

The authors thank the National Cancer Institute for providing the SEER data. We thank TopEdit (www.topeditsci. com) for its linguistic assistance during the preparation of this manuscript.

\section{Author Contributions}

All authors made a significant contribution to the work reported, whether that is in the conception, study design, execution, acquisition of data, analysis and interpretation, or in all these areas; took part in drafting, revising or critically reviewing the article; gave final approval of the version to be published; have agreed on the journal to which the article has been submitted; and agree to be accountable for all aspects of the work.

\section{Funding}

This research did not receive any specific grant from funding agencies in the public, commercial, or not-forprofit sectors.

\section{Disclosure}

The authors report no conflicts of interest in this work.

\section{References}

1. Ewing J. Classics in oncology. diffuse endothelioma of bone. James Ewing. Proceedings of the New York pathological society, 1921. CA Cancer J Clin. 1972;22(2):95-98. doi:10.3322/canjclin.22.2.95

2. Ranft A, Seidel C, Hoffmann C, et al. Quality of survivorship in a rare disease: clinicofunctional outcome and physical activity in an Observational Cohort Study of 618 long-term survivors of Ewing sarcoma. J Clin Oncol. 2017;35(15):1704-1712. doi:10.1200/ jco.2016.70.6226

3. Cotterill SJ, Ahrens S, Paulussen M, et al. Prognostic factors in Ewing's tumor of bone: analysis of 975 patients from the European Intergroup Cooperative Ewing's Sarcoma Study Group. J Clin Oncol. 2000;18(17):3108-3114. doi:10.1200/jco.2000.18.17.3108

4. Esiashvili N, Goodman M, Marcus RB Jr. Changes in incidence and survival of Ewing sarcoma patients over the past 3 decades: surveillance epidemiology and end results data. J Pediatr Hematol Oncol. 2008;30(6):425-430. doi:10.1097/MPH.0b013e31816e22f3

5. Andreou D, Ranft A, Gosheger G, et al. Which factors are associated with local control and survival of patients with localized pelvic Ewing's sarcoma? A retrospective analysis of data from the euroEWING99 trial. Clin Orthop Relat Res. 2020;478(2):290-302. doi:10.1097/corr.0000000000000962

6. Duchman KR, Gao Y, Miller BJ. Prognostic factors for survival in patients with Ewing's sarcoma using the surveillance, epidemiology, and end results (SEER) program database. Cancer Epidemiol. 2015;39(2):189-195. doi:10.1016/j.canep.2014.12.012

7. Leavey PJ, Mascarenhas L, Marina N, et al. Prognostic factors for patients with Ewing sarcoma (EWS) at first recurrence following multi-modality therapy: a report from the Children's Oncology Group. Pediatr Blood Cancer. 2008;51(3):334-338. doi:10.1002/ pbc. 21618

8. Orr WS, Denbo JW, Billups CA, et al. Analysis of prognostic factors in extraosseous Ewing sarcoma family of tumors: review of St. Jude children's research hospital experience. Ann Surg Oncol. 2012;19 (12):3816-3822. doi:10.1245/s10434-012-2458-4

9. Zhou Q, Wu Z-Y, Lin Z-Q. A nomogram to predict prognosis in Ewing sarcoma of bone. J Bone Oncol. 2019;15:100223. doi:10.1016/j.jbo.2019.100223

10. Shi J, Yang J, Ma X, Wang X. Risk factors for metastasis and poor prognosis of Ewing sarcoma: a population based study. J Orthop Surg Res. 2020;15(1):88. doi:10.1186/s13018-020-01607-8

11. Zhang J, Pan Z, Yang J, Yan X, Li Y, Lyu J. A nomogram for determining the disease-specific survival in Ewing sarcoma: a population study. BMC Cancer. 2019;19(1):667. doi:10.1186/ s12885-019-5893-9

12. Wan ZH, Huang ZH, Chen LB. Survival outcome among patients with Ewing's sarcoma of bones and joints: a population-based cohort study. Sao Paulo Med J. 2018;136(2):116-122. doi:10.1590/15163180.2017.0236230917

13. Womer RB, West DC, Krailo MD, et al. Randomized controlled trial of interval-compressed chemotherapy for the treatment of localized Ewing sarcoma: a report from the Children's Oncology Group. J Clin Oncol. 2012;30(33):4148-4154. doi:10.1200/jco.2011.41.5703

14. Chandran R, Kuruva SP, Chennamaneni R, Bala S, Konatam ML, Gundeti S. Outcomes of adult Ewing sarcoma treated with multimodality therapy: a single-institute experience. South Asian J Cancer. 2020;9(4):191-194. doi:10.1055/s-0041-1723108

15. Gupta AA, Pappo A, Saunders N, et al. Clinical outcome of children and adults with localized Ewing sarcoma: impact of chemotherapy dose and timing of local therapy. Cancer. 2010;116(13):3189-3194. doi: $10.1002 /$ cncr.25144

16. Wagenmakers EJ, Farrell S. AIC model selection using akaike weights. Psychon Bull Rev. 2004;11(1):192-196. doi:10.3758/ bf03206482 
17. Linabery AM, Ross JA. Childhood and adolescent cancer survival in the US by race and ethnicity for the diagnostic period 1975-1999. Cancer. 2008;113(9):2575-2596. doi:10.1002/cncr.23866

18. Stiller CA, Passmore SJ, Kroll ME, Brownbill PA, Wallis JC, Craft AW. Patterns of care and survival for patients aged under 40 years with bone sarcoma in Britain, 1980-1994. Br J Cancer. 2006;94(1):22-29. doi:10.1038/sj.bjc.6602885

19. Anderson ND dBR, Young MD, Fuligni F, et al. Rearrangement bursts generate canonical gene fusions in bone and soft tissue tumors. Science. 2018;361(6405). doi:10.1126/science.aam8419

20. Grünewald TG, Bernard V, Gilardi-Hebenstreit P, et al. Chimeric EWSR1-FLI1 regulates the Ewing sarcoma susceptibility gene EGR2 via a GGAA microsatellite. Nat Genet. 2015;47 (9):1073-1078. doi:10.1038/ng.3363

21. Worch J, Matthay KK, Neuhaus J, Goldsby R, DuBois SG. Ethnic and racial differences in patients with Ewing sarcoma. Cancer. 2010;116(4):983-988. doi:10.1002/cncr.24865

22. Davenport JR, Vo KT, Goldsby R, West DC, DuBois SG. Conditional survival and predictors of late death in patients with Ewing sarcoma. Pediatr Blood Cancer. 2016;63(6):1091-1095. doi:10.1002/pbc.25945

23. Hesla AC, Tsagozis P, Jebsen N, Zaikova O, Bauer H, Brosjö O. Improved prognosis for patients with Ewing sarcoma in the sacrum compared with the innominate bones: the Scandinavian Sarcoma Group Experience. J Bone Joint Surg Am. 2016;98(3):199-210. doi:10.2106/jbjs.o.00362
24. Lee J, Hoang BH, Ziogas A, Zell JA. Analysis of prognostic factors in Ewing sarcoma using a population-based cancer registry. Cancer. 2010;116(8):1964-1973. doi:10.1002/cncr.24937

25. Miller BJ, Lynch CF, Buckwalter JA. Conditional survival is greater than overall survival at diagnosis in patients with osteosarcoma and Ewing's sarcoma. Clin Orthop Relat Res. 2013;471(11):3398-3404. doi:10.1007/s11999-013-3147-8

26. Gaspar N, Hawkins DS, Dirksen U, et al. Ewing sarcoma: current management and future approaches through collaboration. J Clin Oncol. 2015;33(27):3036-3046. doi:10.1200/jco.2014.59.5256

27. Brunetto AL, Castillo LA, Petrilli AS, et al. Carboplatin in the treatment of Ewing sarcoma: results of the first Brazilian collaborative study group for Ewing sarcoma family tumors-EWING1. Pediatr Blood Cancer. 2015;62(10):1747-1753. doi:10.1002/pbc.25562

28. Grünewald TGP, Cidre-Aranaz F, Surdez D, et al. Ewing sarcoma Nat Rev Dis Primers. 2018;4(1):5. doi:10.1038/s41572-018-0003-X

29. Friedman DN, Chastain K, Chou JF, et al. Morbidity and mortality after treatment of Ewing sarcoma: a single-institution experience. Pediatr Blood Cancer. 2017;64(11):e26562. doi:10.1002/ pbc. 26562
International Journal of General Medicine

\section{Publish your work in this journal}

The International Journal of General Medicine is an international, peer-reviewed open-access journal that focuses on general and internal medicine, pathogenesis, epidemiology, diagnosis, monitoring and treatment protocols. The journal is characterized by the rapid reporting of reviews, original research and clinical studies

\section{Dovepress}

across all disease areas. The manuscript management system is completely online and includes a very quick and fair peer-review system, which is all easy to use. Visit http://www.dovepress.com/ testimonials.php to read real quotes from published authors. 